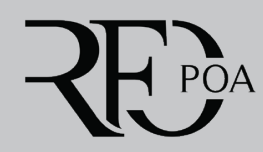

\title{
Promoção da saúde buCAL E o IDOSO
}

\author{
Promotion of bucal health and the elderly
}

\author{
Lucinei Paz Torquato ${ }^{\mathrm{a}}$, (D) Debora Berger Schmidt ${ }^{\mathrm{b}}$
}

\section{RESUMO}

Introdução: Há uma estimativa, para o ano de 2050, de mais de dois bilhões de pessoas acima de 60 anos no mundo. Essa projeção do envelhecimento da população ressalta a importância de avaliar a saúde bucal do idoso e, consequentemente, evidenciar qual o papel das políticas públicas e do cirurgião dentista na promoção de saúde neste contexto. Objetivo: O objetivo deste artigo é abordar as principais alterações bucais que ocorrem no idoso e a importância dada a isso pelas políticas públicas. Discussão: A literatura enfatiza a política nacional de saúde da pessoa idosa e o dever do profissional em promover a qualidade de vida preservando a autonomia e a independência funcional do paciente. Conclusão: Várias políticas públicas garantem os direitos assegurados às pessoas com idade igual ou superior a 60 anos, no que se refere à atenção integral à saúde, porém muito se tem a fazer para que os idosos sejam beneficiados e tenham pleno acesso a ela. Cabe ao cirurgião dentista o despertar para essa realidade e necessidade.

Palavras-chaves: Idoso. Saúde bucal. Promoção da saúde. Doenças da boca.

\section{ABSTRACT}

Introduction: For the year 2050, the estimate is to have more than two billion people over the age of 60 worldwide. This projection of population aging highlights the importance of assessing the oral health of the elderly and, consequently, highlighting the role of public policies and the dental surgeon in health promotion in this context. Objective: The purpose of this paper is to approach the main oral changes that occur in the elderly and the importance given to this by public policies. Discussion: The literature emphasizes the national health policy of the elderly and the duty of the professional to promote the quality of life while preserving the autonomy and functional independence of the patient. Conclusion: Several public policies ensure the rights guaranteed to people aged 60 years or older regarding whole health care for the elderly. However, much needs to be done for them to benefit and have full access to this service. Therefore, it's the dental surgeon's responsibility to bring awareness to this reality and need.

Keywords: Aged. Oral health. Health promotion. Mouth diseases.

\footnotetext{
${ }^{a}$ Cirurgiã dentista da Prefeitura Municipal de Cianorte, Secretaria Municipal de Saúde - Atenção Básica, Unidade Básica de Saúde Lauro Goerll (Cianortinho), Cianorte, PR, Brasil.

b Psicóloga especialista em Atenção Hospitalar, e mestre em Psicologia pela Universidade Federal do Paraná, Pró-Renal Brasil, Curitiba, PR, Brasil.
} Autor de correspondência: Lucinei Paz Torquato - E-mail: lucineitorquato@hotmail.com

Data de envio: 16/04/2019 | Data de aceite: 09/06/2020 


\section{INTRODUÇÃO}

O Estatuto do Idoso traz como obrigação do Estado garantir à pessoa idosa a proteção à vida e à saúde, com políticas públicas efetivas e que permitam um envelhecimento saudável e digno ${ }^{1}$. Também é função das políticas públicas colaborar para que a população idosa tenha um envelhecimento ativo e saudável, permitindo que muitas pessoas cheguem a uma idade mais avançada com o melhor estado de saúde possível. Há uma estimativa, para o ano de 2050, de mais de dois bilhões de pessoas acima de 60 anos no mundo 2 .

A promoção de saúde bucal em idosos busca garantir o bem-estar, a melhoria da qualidade de vida e da autoestima, melhorando a mastigação, estética e possibilidade de comunicação conforme o Caderno de Atenção Básica no $17^{3}$. Ainda segundo esta publicação, as principais condições que comprometem a qualidade de vida do idoso são: cárie de raiz, xerostomia, lesões de tecidos moles, doença periodontal, edentulismo, halitose, dificuldade de higienização, mastigação e deglutição, necessidade de prótese ou uso dela mal adaptada. Desta forma, esta revisão irá abordar as principais alterações bucais que ocorrem no idoso e a importância dada a isso pelas políticas públicas, salientando também o dever do cirurgião dentista frente à promoção da saúde bucal do idoso.

\section{REVISÃO DE LITERATURA}

\section{Políticas públicas e a saúde bucal do idoso}

No Brasil e no mundo, a população de idosos tem aumentado de forma acelerada e intensa ${ }^{4}$. Não se pode negar a importância da promoção da saúde neste processo e, de modo particular, reconhecer que a promoção de saúde bucal, ao longo da vida, contribui para que tal população chegue a essa fase com bem-estar integral ${ }^{5}$. É necessário o desenvolvimento de estudos sobre esta temática que subsidiem a disseminação de informação, sensibilização e mudanças de hábitos e condutas, as quais são necessárias para a assertividade das ações e positividade dos resultados de promoção da saúde bucal. A relevância do tema foi abordada na $1^{\text {a }}$ Conferência nacional de saúde bucal, cujo relatório final menciona:

A saúde bucal é parte integrante e inseparável da saúde geral do indivíduo e está relacionada diretamente com as condições de saneamento, alimentação, moradia, trabalho, educação, renda, transporte, lazer, liberdade, acesso e posse da terra, aos serviços de saúde e a informação ${ }^{6}$.

Os direitos das pessoas com idade igual ou superior a 60 anos estão previstos no Estatuto do Idoso, através da lei 10.741 de 1 outubro de $2003^{1}$. Dentre os direitos, assegura a atenção integral a saúde do idoso por intermédio do Sistema Único de Saúde (SUS), garantindo-lhes o acesso universal e igualitário, em conjunto articulado e contínuo com as ações e serviços, para a prevenção, promoção, proteção e recuperação da saúde, incluindo atenção especial as doenças que afetam preferencialmente os idosos ${ }^{1}$. Este Estatuto, não só reafirma todos os direitos fundamentais próprios da pessoa idosa como, destaca o direito à saúde como um dever do Estado e da sociedade, enfocando também a saúde bucal, pois, embora não citado pelo legislador, a Odontologia e a Odontogeriatria fazem parte da Gerontologia ${ }^{7}$. Somado a isso, Smeltzer e Bare ${ }^{\mathrm{i}}$ apud Przylynski et al. ${ }^{8}$ referem que o sorriso revela alegria, segurança e satisfação em relação ao outro, ou seja, que a saúde bucal além de ser parte integrante e importante da saúde geral, é um fator determinante na qualidade de vida, autoestima e contato social.

\footnotetext{
i Smeltzer SC, Bare BG. Tratado de enfermagem médico - cirúrgica. Rio de Janeiro: Guanabara Koogan; 2002.
} 
Apesar disto, a oferta de serviços odontológicos aos idosos na área pública do Brasil ainda é restrita. O principal motivo para os idosos não procurarem o serviço odontológico é não perceber tal necessidade ${ }^{9}$. O fato de muitas doenças detectadas ao exame clínico serem assintomáticas e desconhecidas ao indivíduo seria, de acordo com Locker e Jokovic ${ }^{10}$, uma das razões para a fraca associação entre a realidade clínica e a auto avaliação do indivíduo, este que, muitas vezes, acha sua condição favorável mesmo em condições insatisfatórias ${ }^{9}$. Para Mello e Erdamann ${ }^{11}$, nem sempre o idoso entende o que, para o profissional, seja uma urgência. Assim, o comportamento do idoso será moldado de acordo com a sua própria percepção e importância, podendo colocar em vulnerabilidade a sua condição de saúde bucal' ${ }^{12}$ :

A condição de saúde bucal é determinada, mas também determina os comportamentos em saúde bucal. Os comportamentos de saúde bucal referem-se as práticas pessoais como escovação, uso de fio dental, hábitos tabagistas e uso formal de serviços odontológicos preventivos, curativos e reabilitadores ${ }^{13}$.

Embora a capacidade de adquirir novas habilidades tenda a diminuir no idoso, vale ressaltar que ele pode possuir potencial de aprendizagem. Para isso, é importante valorizar o que já é de conhecimento dos idosos, suas necessidades e seus interesses ${ }^{\mathrm{i} \text { apud } 8}$.

A epidemiologia evidencia que o quadro de saúde bucal da população idosa brasileira é precário, ao passo que não há programas efetivos voltados para esse grupo populacional, em que o atendimento não é adequado ${ }^{14}$. Com o crescimento rápido da população idosa brasileira, a sociedade e, principalmente, os sistemas de saúde sofrem um impacto. A infraestrutura em termos de instalações, programas específicos, recursos humanos com qualidade e quantidade para responder a essa demanda é precária e insuficiente ${ }^{5}$.

Em 2003, foi divulgado o relatório do projeto Saúde Bucal (SB Brasil, 2003) que evidenciou como alta a experiência de cárie e perda dentária de idosos brasileiros e, com isso, a necessidade de implementação de programas educadores e preventivos pelo serviço público ${ }^{14}$. Para Lucena et al. ${ }^{15}$, a Política Nacional de Saúde Bucal - Brasil Sorridente, constitui um marco histórico das políticas públicas, que agrega discussões desde o Movimento pela Reforma Sanitária Brasileira, além de apresentar em seus pressupostos os princípios do Sistema Único de Saúde. O primeiro programa desenvolvido pelo Ministério da Saúde, em 2004, foi o Brasil Sorridente, com objetivo de organizar atenção, ampliar e garantir o acesso da população brasileira à saúde bucal. Dentre outras ações, trouxe os Centros de Especialidades Odontológicas (CEO) e Laboratórios Regionais de Próteses Dentárias (LRPD), garantindo, também, na assistência à saúde, atendimento especializado e integralizado ${ }^{16}$.

Os profissionais de saúde devem garantir o acolhimento da pessoa idosa, compreender as particularidades dessa população e a legislação; devem para isso, estar preparados para as questões do processo de envelhecimento e, principalmente, no que se refere à subjetividade da pessoa idosa ${ }^{2}$. Além de garantir o acesso e motivar o uso entre idosos dentados e edentados, é fundamental esclarecer, principalmente para os edentados, a necessidade e a importância do uso de serviços odontológicos, pois observa-se uma tendência ao uso somente quando a situação da saúde bucal é crítica, ou seja, na presença de dor ou quando a aparência é percebida como péssima ou ruim ${ }^{13}$.

O fácil acesso aos serviços odontológicos e uma equipe de cuidadores conscientes da importância de uma boa condição bucal são recursos para a manutenção da autonomia do indivíduo idoso ${ }^{17}$. De acordo com Bulgarelli e $\mathrm{MançO}^{18}$, observa-se que políticas públicas já deram início à atenção integral à saúde do idoso, porém muito se tem a fazer para que os idosos sejam beneficiados e tenham pleno acesso a ela. Nos dias atuais, o Brasil vive um processo de reorganização e adequação da atenção básica voltada a saúde bucal.

Contudo, essas ações ainda representam uma atenção fragmentada e longe do que é previsto nas políticas de saúde públicas aos idosos ${ }^{19}$. Além disso, adiciona-se o fato de que, para reorganizá-la requer um planejamento inter e multidisciplinar ${ }^{20}$. 


\section{Principais alteraçõES BUCAIS NO IDOSO}

A queixa do paciente representa o principal motivo que o leva a procurar um serviço de saúde bucal e pode apresentar alterações de uma possível patologia ${ }^{18}$. O cirurgião dentista precisa ter conhecimento sobre as condições de saúde bucal dos idosos, e como elas podem afetar sua condição sistêmica ${ }^{14}$. Afinal, a saúde bucal comprometida pode afetar o nível nutricional, o bem-estar físico e mental e diminuir o prazer de uma vida social ativa ${ }^{21}$. Idosos apresentam alterações fisiológicas e patológicas que acometem o organismo, bem como também o uso de alguns medicamentos que podem apresentar efeitos colaterais e até mesmo alterações no nível de saúde geral ${ }^{4}$.

Algumas enfermidades comuns ao paciente idoso, como o câncer, a artrite, o diabetes e o mal de Parkinson apresentam consequências bucais que interferem no tratamento odontológico. Por exemplo, os pacientes com artrites apresentam habilidade manual comprometida para realização da higiene bucal ${ }^{4}$. Kandelman et al. ${ }^{22}$ cita que entre os idosos são frequentes doenças como é o caso da demência e da doença de Alzheimer, nas quais a diminuição na destreza manual interfere com o estado de saúde oral ${ }^{23}$.

As principais alterações e doenças que se manifestam no idoso, como: redução da capacidade gustativa, língua saburrosa, varicosidades linguais, xerostomia, síndrome de ardência bucal, fibrosa inflamatória, traumatismos crônicos, candidose, úlcera da queilite angular, líquen plano, herpes, leucoplasias, queilite actínica, carcinoma espinocelular e outros $^{24}$. Segundo Barbosa ${ }^{14}$, conhecer a condição de saúde bucal dos idosos é um meio de identificar os problemas bucais, onde e como intervir e de que forma os programas sociais podem atuar na promoção e prevenção da saúde bucal.

São atribuições do cirurgião dentista para com essa população: realizar atenção integral, solicitar exames complementares necessários, prescrever medicamentos, realizar assistência domiciliar além de orientar a pessoa idosa, seus familiares e/ou o cuidador sobre a importância da higienização da boca e da prótese ${ }^{2}$. Para Roncalli et al. ${ }^{\text {ii }}$ apud Moreira et al..$^{25}$, a prática histórica de uma odontologia mutiladora é rompida quando uma nova lógica assistencial é introduzida através de assistência com base no domicílio ${ }^{25}$.

Para Rocha e Miranda ${ }^{26}$, o atendimento odontológico domiciliar para idosos é uma estratégia educativa, assistencial e multidisciplinar que tem como objetivo intervir no processo saúde-doença. Nesta modalidade de atendimento, o cirurgião dentista deve dar maior atenção aos principais responsáveis, cuidadores e familiares, pela realização das medidas de higiene bucal dos idosos dependentes ${ }^{26}$. O cuidador do idoso desempenha tarefas que precisam de acompanhamento por pessoas capacitadas, podendo assim, manter o bem-estar do idoso ${ }^{27}$. Além disso, para Mello e Padilha ${ }^{28}$ a percepção do cuidador, para com sua saúde bucal, influencia no cuidado que ele tem para com o idoso, sendo comum que transfira para este as mesmas ações que tem para consigo.

Os idosos brasileiros de hoje mostram um perfil que é fruto de um modelo de atenção de pior acesso e a extração dentária era o que predominava. Porém, os futuros idosos do Brasil terão melhor preservação de seus dente ${ }^{16}$. A perda dentária também é consequência da falta de acesso e da não utilização de serviços públicos odontológicos e de atitudes errôneas dos profissionais da odontologia ${ }^{29}$. Há uma crença no Brasil de que é normal perder dente com avanço da idade ${ }^{30}$. Com vistas a diminuição de tal crença, a odontologia tem desenvolvido ações de promoção a saúde bucal e oferecido a população idosa várias soluções para perdas dentarias, sendo mais comuns as próteses ${ }^{30}$. Ainda no Estatuto do Idoso, alguns itens estão

\footnotetext{
ii Roncalli AG, Arcieri RM, Unfer B, Costa ICC, Moraes E, Saliba NA. Modelos assistenciais em saúde bucal no Brasil: tendências e perspectivas. Ação Coletiva 1999;2:9-14.
} 
diretamente relacionados ao atendimento odontológico, como por exemplo, o fornecimento gratuito de medicamentos, próteses e órteses ${ }^{1}$.

Na linha guia de saúde bucal do Paraná, as próteses dentárias fazem parte de procedimentos da atenção primária. Cabe ao profissional realizar desde moldagem até monitoramento do paciente ao longo do tempo ${ }^{29}$. Controlar o ambiente bucal é uma medida preventiva específica e importante para os idosos, principalmente para a prevenção de cáries dentais, periodontopatias e infecções oportunistas como candidíase ${ }^{31}$. Traumas, patologias, hábitos orais ou ainda disfunção de glândulas salivares podem ser causas de alterações na mucosa oral $^{23}$. O câncer de boca mais frequente é o carcinoma espinocelular. Dentre os canceres humanos, o de boca ocupa o terceiro lugar. É fundamental o papel do cirurgião dentista na detecção de qualquer anormalidade que o idoso apresenta na cavidade bucal ${ }^{24}$.

No Paraná, a Secretaria de Saúde Estadual tem como proposta o uso de azul de toluidina a $1 \%$ associado ao acido acético a 1\% para um rastreamento do câncer bucal, fortalecendo assim o envolvimento do cirurgião dentista ao diagnóstico precoce com método simples que pode aumentar a sobrevida do paciente. Lembrando que a falta de diagnóstico precoce tem a ver com pouco preparo do profissional e deficiente acesso da população idosa ${ }^{29}$.

De certa forma, resultados positivos de programas de saúde não dependem somente de ações específicas, sendo necessário que a população saiba para querer, e queira para pedir e fazer. A população precisa se dispor a ter uma postura ativa, assimilando hábitos e modos de vida saudável, perceber suas necessidades de saúde e desenvolver o autocuidado ${ }^{31}$.

\section{Discussão}

\section{O cirurgião dentista frente a promoção de saúde bucal do idoso}

para manter o status de saúde dos idosos é importante investir em programas de promoção envolvendo saúde bucal e sistêmica ${ }^{14}$, recomendando-se que as ações de promoção de saúde sejam intersetoriais e interdisciplinares. O Instituto Nacional do Câncer (INCA) recomenda dieta saudável rica em frutas e verduras como prevenção contra o câncer. Igualmente, há a recomendação para a prática de atividades físicas, pois o sedentarismo pode prejudicar a dieta e trazer má-nutrição para os idoso ${ }^{16}$. Além de vários estudos trazerem uma relação da doença periodontal e cardiovascular, inclusive ${ }^{16,32,33}$. A política nacional de saúde bucal norteia também para a necessidade de ampliar o acesso de atenção as doenças por linhas de cuidado e condição de vida e recomenda a organização e desenvolvimento de ações de prevenção e controle do câncer bucal ${ }^{34}$.

É válido citar que, em 2006, na perspectiva de superar dificuldades, o SUS implantou o Pacto pela Saúde, constituído por 3 componentes: pacto pela vida, pacto em defesa do SUS e pacto de gestão do SUS. Com o objetivo de implantar a Política Nacional de Saúde da Pessoa Idosa, o pacto pela vida, dentre outras prioridades, tem como diretriz a atenção integral a saúde da pessoa idosa. Ainda propõe o Programa de Educação Permanente à distância voltado para profissionais que trabalham na rede de atenção básica em saúde, contemplando os conteúdos específicos das repercussões do processo de envelhecimento populacional para a saúde individual e para a gestão dos serviços de saúde ${ }^{35}$.

Para Côrte-Real et $a .^{23}$, as principais causas para o crescimento demográfico da população idosa são as melhorias nas condições de vida, cuidados de saúde e políticas públicas de saúde. Ainda que o acesso seja, por vezes, limitado por diversos fatores - econômicos, médicos e psicossociais.

Cabe aos profissionais da Odontologia conhecer o processo de envelhecimento e estabelecer ações de promoção à saúde, bem como reabilitação estomatognática coerente a fim 
de manter e recuperar a saúde bucal ${ }^{14}$. Ainda que a Odontogeriatria seja reconhecida como uma especialidade voltada aos idosos, muito deve ser feito em relação ao preparo dos futuros dentistas, considerando a realidade privada e principalmente pública ${ }^{4}$.

É dever de todo aquele que trata com ser humano e que de alguma forma tenha possibilidade de vê-lo, palpá-lo, senti-lo, enfim, que tenha a oportunidade de examiná-lo, assegurar a sanidade e higidez dos tecidos bucais, procurando alterações mínimas, insignificantes e inócuas aparentemente e tratá-las, a fim de que não se tornem mais um número na estatística dos mortos de câncer de boca ${ }^{24}$.

De acordo com a Política Nacional de Saúde da Pessoa Idosa, todo profissional, quando chamado a atender o idoso, deve procurar promover a qualidade de vida. Preservando a autonomia e a independência funcional como meta em todos níveis de atenção. Dentre os eixos, dois são muito importantes: enfrentar a fragilidade do idoso, família e sistema de saúde; e promover a saúde e integração social ${ }^{36}$. A Odontologia deve atuar como um agente nesta integração, mantendo a saúde bucal do idoso, favorecendo uma aparência agradável e melhor autoestima ${ }^{17}$. Um exemplo de promoção de saúde e integralidade é o Projeto de Extensão "Promoção de Saúde Bucal para pacientes da Terceira Idade" da Universidade Estadual de Londrina - PR, onde o paciente é informado sobre os cuidados com a saúde bucal, a comunidade é conscientizada sobre a importância da prevenção de doenças bucais e com isso redução da incidência das doenças dos idosos. Podendo ser aplicado em qualquer outro lugar e principalmente, favorecendo a integralidade entre servidores e a comunidade ${ }^{37}$.

\section{CONCLUSÃo}

Esta pesquisa de revisão bibliográfica foi realizada com o objetivo de servir como base para conscientização da importância da saúde bucal nos idosos, evidenciando as principais alterações bucais e o papel do cirurgião dentista para a prevenção e promoção da saúde bucal. Os estudos evidenciaram que muitas políticas públicas garantem os direitos assegurados as pessoas com idade igual ou superior a 60 anos. No que se refere à atenção integral à saúde do idoso, porém, muito se tem a fazer para que os idosos sejam beneficiados e tenham pleno acesso a ela. Portanto, o cirurgião dentista deve despertar para essa realidade e necessidade, buscando rever a problemática, buscando cooperar com o desenvolvimento de ações e mecanismos que reestruturem a prática da prevenção e promoção da saúde bucal.

\section{REFERÊNCIAS}

1. Brasil. Ministério da Saúde. Secretaria de Atenção à Saúde. Departamento de Atenção Básica. Estatuto do Idoso. Brasília, DF; 2003

2. Brasil. Ministério da Saúde. Secretaria de Atenção à Saúde. Envelhecimento e saúde da pessoa idosa. Brasília, DF: Ministério da Saúde; 2006. 192 p. (Série A. Normas e manuais técnicos); (Cadernos de Atenção Básica; n. 19).

3. Brasil. Ministério da Saúde. Secretaria de Atenção à Saúde. Saúde bucal. Brasília, DF: Ministério da Saúde; 2008. 92 p. (Série A. Normas e manuais técnicos); (Caderno de Atenção Básica; n. 17).

4. Rosa LB, Zuccolotto MCC, Batglion C, Coronatto E. Odontogeriatria: a saúde bucal na terceira idade. RFO UPF. 2010;13(2):82-6.

5. Mello ALSF, Erdmann AL. O envelhecer ativo e os desafios para a odontogeriatria na promoção a saúde. Rev RENE. 2003;4(2):103-11.

6. Brasil. Ministério da Saúde. Secretaria de Atenção à Saúde. Coordenação Nacional de Saúde Bucal. $1^{\text {a }}$ Conferência nacional de saúde bucal: relatório final. [Internet]. Brasília, DF: 1986.11p. [acesso 2019 abril 12]. Disponível em: https:// aps.saude.gov.br/biblioteca/index.

7. Hebling E, Rodrigues CK. O estatuto do idoso e a saúde bucal. Robrac. 2006;15(39):51-6. 
8. Przylynski DS, Pelzer MT, Santos SSC, Silva ME, Costa CFS, Gasparim AB. Ações educativas de enfermagem em saúde bucal de idosos em uma instituição de longa permanência. Cog Enf. 2009;14(4):696-702.

9. Silva SRC da, Fernandes RAC. Auto percepção das condições de saúde bucal por idosos. Rev Saúde Pública. 2001;35(4):349-55.

10. Locker D, Jokovic A. Using subjective oral health status indicators to screen for dental care needs in older adults. Community Dent Oral Epidemiol. 1996;24:398-402.

11. Mello ALSF, Erdamann AL. Revelando contradições e incorporando melhores práticas no cuidado à saúde bucal de idosos. Physis. 2007;17(1):139-56.

12. Martins A, Barreto SM, Silveira MF, Santa-Rosa TTA, Pereira SM. Autopercepção da saúde bucal entre idosos brasileiros. Rev Saúde Pública. 2010;44(5):912-22.

13. Martins AME de BL, Barreto SM, Pordeus IA. Características associadas ao uso de serviços odontológicos entre idosos dentados e edentados no Sudeste do Brasil: Projeto SB Brasil. Cad Saúde Púb. 2008;24(1):81-92.

14. Barbosa KGN. Condições de saúde bucal em idosos: uma revisão da realidade brasileira. Odontol Clín-Cient. 2011 Jul/ Set;10(3):227-31.

15. Lucena EHG, Pucca Junior GA, Sousa MF. A política nacional de saúde bucal no Brasil no contexto do Sistema Único de Saúde. Tempus (Brasília). 2011;5(3):53-63.

16. Vargas AMD, Vasconcelos M, Ribeiro MTF. Saúde bucal: atenção ao idoso. Belo Horizonte: Nescon/UFMG; 2011.

17. Araújo SSC de, Freire DB de L, Padilha DMP, Baldisserotto ]. Suporte social, promoção de saúde e saúde bucal na população idosa no Brasil. Interface (Botucatu). 2006;10(19):203-16.

18. Bulgarelli AF, Manço ARX. Saúde bucal do idoso: revisão. Clin Pesq Odontol. 2006 Abr/Jun;2(4):319-26.

19. Piuvezam G, Lima KC de, Carvalho MS de, Xavier VGP, Silva RA da, Dantas ARF, et al. Atenção primária à saúde e os idosos institucionalizados: a perspectiva da gestão municipal no Brasil. Rev. Port Saúde Púb. 2016;34(1):92-100.

20. Dutra CESV, Sanchez HF. Organização da atenção à saúde bucal prestada ao idoso nas equipes de saúde bucal da Estratégia Saúde da Família. Rev Bras Geriatr Gerontol. [Internet]. 2015;18(1):179-88. [acesso 2019 out 26]. Disponível em: http://dx.doi.org/10.1590/1809-9823.2015.13184.

21. Werner CW, Saunders M], Paunovich E, Yeh C. Odontologia geriátrica. Rev Fac Odontol Lins. 1998;11(1):62-9.

22. Kandelman D, Petersen PE, Ueda H. Oral health, general health, and quality of life in older people. Spec Care Dentist. 2008;28:224-36

23. Côrte-Real IS, Figueiral MH, Campos JCR. As doenças orais no idoso: considerações gerais. Rev Port Estomatol Med Dent Cir Maxilofac. 2011]ul/Set;52(3):175-80.

24. Silva EMM da, Barao VAR, Santos DM, Delben ]A, Ribeiro ACP, Gallo, AKG. Principais alterações e doenças bucais que acometem o paciente geriátrico: revisão de literatura. Odont. 2011;19(37):39-47.

25. Moreira RS, Nico LS, Tomita NE, Ruiz T. A saúde bucal do idoso brasileiro: revisão sistemática sobre o quadro epidemiológico e acesso aos serviços de saúde bucal. Cad Saúde Púb. 2005;21(6):1665-75.

26. Rocha DA, Miranda AF. Atendimento odontológico domiciliar aos idosos: uma necessidade na prática multidisciplinar em saúde: revisão de literatura. Rev Bras Geriatr Gerontol. 2013;16(1):181-9.

27. Saliba NA, Moimaz SAS, Marques JAM, Prado RLI. Perfil de cuidadores de idosos e percepção sobre saúde bucal. Interface. 2007:11(21):39-50.

28. Mello ALF, Padilha DMP. Instituições geriátricas e negligência odontológica. Rev Fac Odontol Porto Alegre. 2000;41(1):44-8.

29. Paraná. Secretaria de Estado da Saúde do Paraná. Superintendência de Assistência à Saúde. Linha Guia Rede de Saúde Bucal. Curitiba; 2016.

30. Cardoso MCAF, Roseneide VB. A saúde bucal e as funções da mastigação e deglutição nos idosos. Stud Interdiscipl Envelhec. 2010;15(1):53-67.

31. Shinkai RSA, Cury AADB. O papel da odontologia na equipe interdisciplinar: contribuindo para a atenção integral ao idoso. Cad Saúde Pública. 2000;16(4):1099-109.

32. Vieira WR. Doença cardiovascular e doença periodontal. Rev Bras Cir Cardiovasc. 2014;29(1):VII-IX.

33. Saldanha KFD, da Costa DC, Masocatto DC, Oliveira MM, Gaetti Jardim EC. Doença periodontal e doenças cardiovasculares: revisão de literatura. Arch Health Invest. 2015;4(1):31-6.

34. Brasil. Ministério da Saúde. Diretrizes da política nacional de saúde bucal. Brasília, DF: Ministério da Saúde; 2004.

35. Brasil. Ministério da Saúde. Portaria n³99/GM, de 22 fevereiro de 2006. Divulga o pacto pela saúde 2006-Consolidação do SUS e aprova as diretrizes operacionais do referido pacto. Diário Oficial [da] república Federativa do Brasil, Poder Executivo, Brasília, DF, 23 fev. 2006. Seção 1, p. 43-51.

36. Brasil. Ministério de Saúde. Portaria n²528/GM, de19 outubro de 2006. Aprova a política nacional de saúde da pessoa idosa. Diário Oficial [da] república Federativa do Brasil, Poder Executivo, Brasília, DF, 20 out. 2006. Seção 1, p. 142.

37. Barbosa CS, Silva LAMP da, Ferraresso F de OT, Higasi MS, Cassemiro SS. Promoção de saúde bucal para paciente da terceira idade. [Internet]. In: Anais $37^{\circ}$-SEURS Seminário de Extensão Universitário da Região Sul Florianópolis: Repositório Institucional da UFSC; 2019. [acesso 2019 out 26]. Disponível em: https://repositório.ufsc.br/handle/123456789/199285. 